\title{
Mixing material modes
}

\author{
S.C. Pont*a, J.J. Koenderink ${ }^{b}$, A.J. van Doorn ${ }^{a}$, M.W.A. Wijntjes ${ }^{a}$, S.F. te Pas ${ }^{c}$ \\ ${ }^{a}$ Perceptual Intelligence lab, Industrial Design Engineering, Delft University of Technology, \\ Landberstraat 15, Delft, the Netherlands; \\ ${ }^{b}$ Perceptual Intelligence lab, Electrical Engineering Mathematics and Computer Science, Delft \\ University of Technology, Mekelweg 4, Delft, the Netherlands; \\ ${ }^{c}$ Experimental Psychology, Helmholtz Institute, Utrecht University, Heidelberglaan 2, Utrecht, the \\ Netherlands
}

\begin{abstract}
We present a novel setup in which real objects made of different materials can be mixed optically. For the materials we chose mutually very different materials, which we assume to represent canonical modes. The appearance of 3D objects consisting of any material can be described as linear superposition of 3D objects of different canonical materials, as in "painterly mixes". In this paper we studied mixtures of matte, glossy and velvety objects, representing diffuse, forward and asperity scattering modes.

Observers rated optical mixtures on four scales: matte-glossy, hard-soft, cold-warm, light-heavy. The ratings were done for the three combinations of glossy, matte, and velvety green birds. For each combination we tested 7 weightings.

Matte-glossy ratings varied most over the stimuli and showed highest (most glossy) scores for the rather glossy bird and lowest (most matte) for the rather velvety bird. Hard-soft and cold-warm were rated highest (most soft and warm) for rather velvety and lowest (most hard and cold) for rather glossy birds. Light-heavy was rated only somewhat higher (heavier) for rather glossy birds. The ratings varied systematically with the weights of the contributions, corresponding to gradually changing mixtures of material modes. We discuss a range of possibilities for our novel setup.
\end{abstract}

Keywords: Material perception, qualities, ecological optics, appearance, imaging, HDR, glossiness

\section{INTRODUCTION}

Natural materials cover a wide range of mutually very different optical properties ${ }^{1,4}$. However, it has been suggested ${ }^{4}$ that they can be categorized into roughly a dozen types of canonical modes on the basis of their bidirectional surface scattering or BRDF (for this paper we do not consider spatial texture). The lobes of the Bidirectional Reflectance Distribution Function may be described by their average direction, e.g. in the illumination direction (backscattering or retroreflection ${ }^{7}$ ), roughly in the specular direction (forward scattering or specular reflection ${ }^{8}$ ), distributed over all directions (diffuse scattering ${ }^{5}$ ), along the surface (asperity scattering ${ }^{3}$ ) or perpendicular to it. This primary scattering direction is associated with certain visual characteristics or modes of material appearance, for instance forward scattering with glossy-/shiny-/specularness and diffuse scattering with matteness. The BRDF of generic materials typically consist of several of such lobes, e.g. a peach BRDF may contain a diffuse and a surface scattering lobe, resulting in a combination of a matte and a velvety mode. Since the primary scattering direction is different for these modes, the salient features of the appearance modes usually show up at different locations too. Thus, similarly to descriptions of BRDFs as linear superpositions of scattering lobes, we can describe the appearance of 3D objects consisting of any materials as linear superpositions of 3D objects of different canonical materials, e.g. a glossy object as the superposition of a specular and a matte object.

*s.c.pont@tudelft.nl; phone +31 (0)15 273243; fax +31 $\quad(0) 15 \quad 2787179 ; \quad$ pi-lab.nde-lab.nl/home.html \& www.sylviapont.nl

Human Vision and Electronic Imaging XVII, edited by Bernice E. Rogowitz, Thrasyvoulos N. Pappas,

Huib de Ridder, Proc. of SPIE-IS\&T Electronic Imaging, SPIE Vol. 8291, 82910D • (C) 2012 SPIE-IS\&T

CCC code: $0277-786 \mathrm{X} / 12 / \$ 18 \cdot$ doi: $10.1117 / 12.916450$ 
We are particularly interested in the perception of material qualities that are important for interaction with objects. Mausfeld ${ }^{6}$ discussed such qualities from a theoretical point of view, but did not list relevant perceptual properties explicitly. The only empirically based inventory of such qualities that we are aware of, was made by Karana ${ }^{2}$. She inventorized perceptual properties of materials that are important for interacting with industrial products. She found the following list: color intensity, colorfulness, ductility, elasticity, glossiness, softness, odour, reflectiveness, roughness, strength, transparency, warmth and heaviness. A few of these terms are ambiguous to us, e.g. color intensity and reflectiveness. Also, the list is probably not complete, e.g. we miss terms such as glitter, luminosity, regularity (of texture). Furthermore, Karana treats color as a separate object property that interacts with the perceptual properties in determining the meaning of materials. We distinguish the physical spectral scattering properties of an object and its color (a perceptual property by definition). Although we would have formulated a slightly different set of properties, we think that Karana's inventory provides a sufficient, practical, empirical basis. Therefore we will use this list as a rough guideline to select our rating dimensions. Such perceptual material properties determine the overall impression or quality of a material, for instance the smoothness, hardness and glossiness of car lacquers or the elasticity, softness, warmth and lightness of a shirt. The quality of the material interacts with form in determining the meanings of materials, e.g. the lacquers on a racecar or a family car may be associated with different meanings.

In this study we chose a matte, a glossy, and a velvety object as basic, canonical materials. These materials represent diffuse, forward and asperity scattering modes. We chose these materials, because they represent modes that are mutually very different (in terms of BRDF), often encountered in natural scenes (e.g. in plastic, paper, furniture, cloths, plant leaves) and because of practical reasons: it was possible to finish objects with these materials using matte and glossy paints and having it flocked. The bird was chosen for its shape and size: the local shape varied over the object, but was not too complicated to spray paint or flock it homogeneously, and fitted well in our setup. The finishes were done in a color, green, such that color differences between scattering characteristics would be salient; highlights and bright contours due to asperity scattering reflect the primary lighting while diffuse scattering reflects the body color.

In our experiment we studied a selection of properties from Karana's list ${ }^{2}$, namely glossiness, hardness, warmth and lightness. We chose these properties, because we wanted to test: 1) at least one visual property with an obvious and clear relation to our visual stimuli (glossiness should be rated high for our glossy object and low for the matte object, and ratings should systematically vary for the optical mixtures), and 2) a few other properties that are especially important for interaction and overall quality, but without a clear relation to optical material properties. Since we chose to study mixtures of glossy, matte and velvety modes of an opaque, green bird of constant shape, we decided to also test softness, warmth and heaviness perception. In our experiment observers rated these qualities on matte-glossy, hard-soft, coldwarm and light-heavy scales. Our experiment will provide insights into how combinations of optical material modes are related to perceived material qualities. Our main question in this experiment was whether gradually varying mixtures would result in gradually and systematically varying judgments. Secondly, we wanted to know which range of weightings is reasonable under natural viewing conditions and whether very small contributions might still influence material perception.

\section{EXPERIMENT}

\subsection{Methods}

\section{Setup and stimuli}

We made a setup to optically mix two objects in a linearly weighted manner, see figure 1. It consists of a black square box with $30 \mathrm{~cm}$ ribs in which we put a vertical semi-transparent optical mirror. The mirror was placed diagonally in the box, that is, at 45 degrees with respect to the viewing direction. One of the objects was seen through the mirror. The other object was seen via the reflection in the mirror. The objects were lined up symmetrically with respect to the mirror to superpose them. The illumination apertures consisted of $6 \mathrm{~cm}$ by $6 \mathrm{~cm}$ square openings in the top of the box, under which we affixed $6 \mathrm{~cm}$ long light baffles in order to prevent light from one side of the mirror to reach the object on the opposite side. The box was put in a Ganzfeld (fully diffuse light field). The stimuli in the box are viewed by way of a 50 $\mathrm{cm}$ long $15 \mathrm{~cm}$ wide black tube. There was a shutter in front of the tube that could be lifted by the observer in order to view the stimulus. There was a plastic wall between the viewing hole / observers and the setup / experimenter to prevent the participants from seeing the setup, stimuli and settings.

The stimuli were optical mixtures of three objects of the same shape but finished with different materials: birds with matte green paint, with glossy green paint and with a green flock finish (the matte, glossy and velvety bird, respectively), 
see figure 2. The green color was matched via the RAL system (the matching is messed up in the photography, in the setup the colors did match to the human eye). The luminance of the two objects was equalized; to this aim we had to attenuate the contribution from behind the mirror with a 0.5 neutral density filter (NDF). The weights of the two contributions were varied by way of neutral density filters (NDF's) of various densities over the lighting openings in the box. We ran psychophysical experiments in the equalized condition and with NDFs of $0.5,1$ and 2 , resulting in 7 weighted combinations in which the contributions were weighted equally or in which one of the contributions was attenuated by a factor of $10^{0.5}, 10^{1}$ or $10^{2}$. These 7 weightings were tested for each of the three material pairs, thus we obtained 21 conditions altogether.

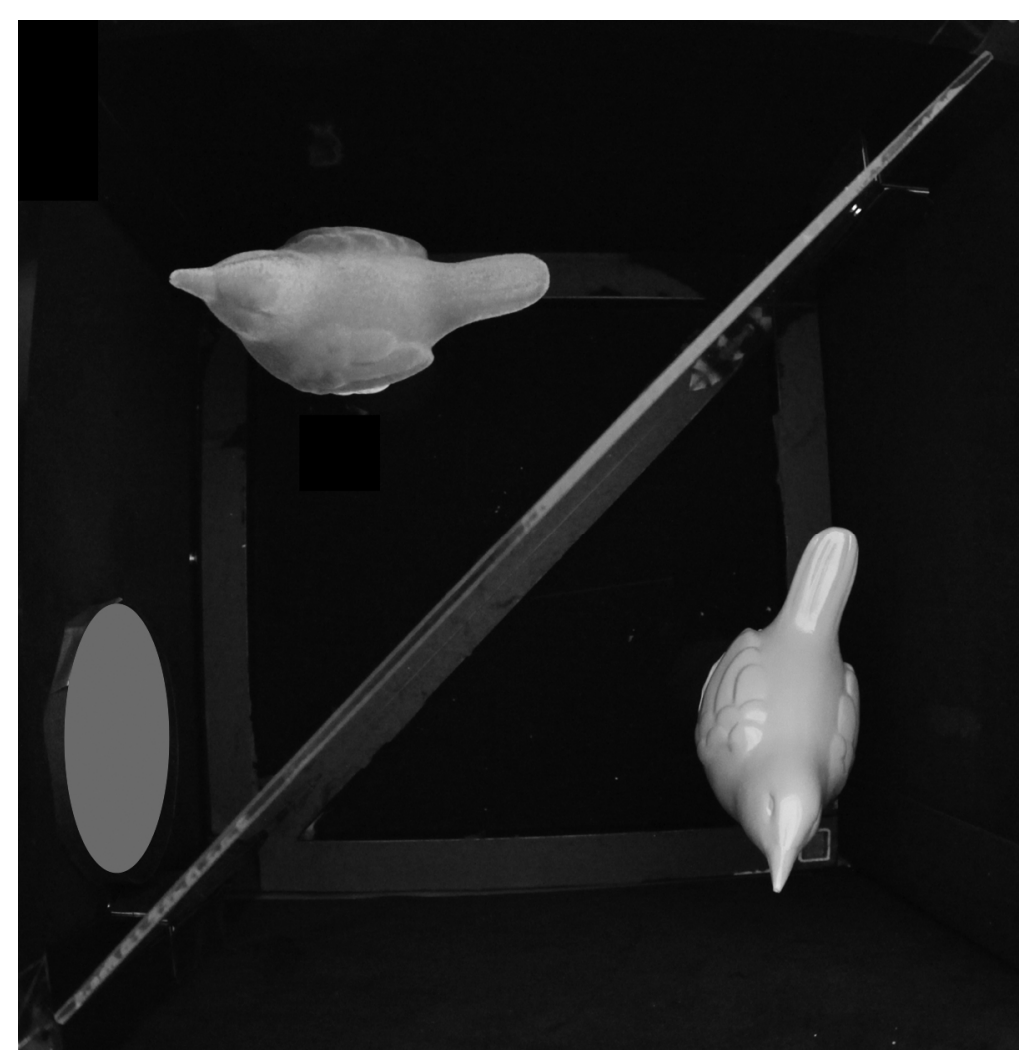

Figure 1. A topview of the inner part of our setup. The setup consists of a box of $30 \times 30 \times 30 \mathrm{~cm}$, of which the inside is covered by black flocked paper. A semi-transparent mirror was placed vertically at the diagonal of the box - the reflection at the upper side of the mirror is clearly visible. The gray ellipse depicts the viewing hole in the box (since the setup is viewed through a tube made of black flocked paper it was not visible in the photograph).

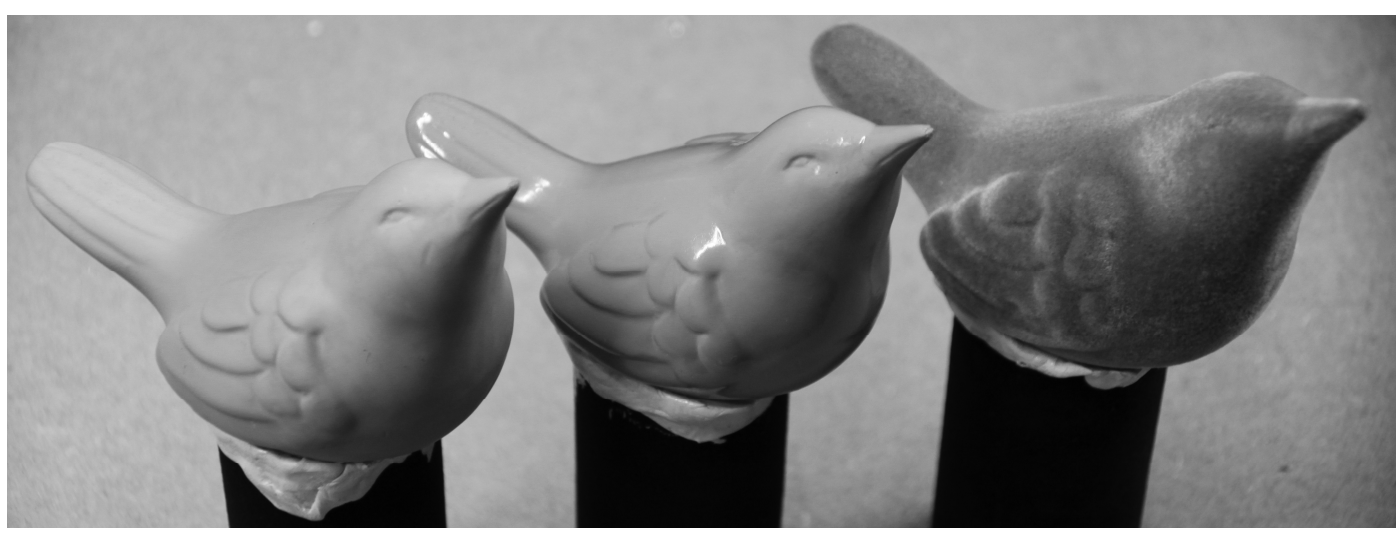

Figure 2. The matte (left), the glossy (centre), and the velvety (right) birds. 


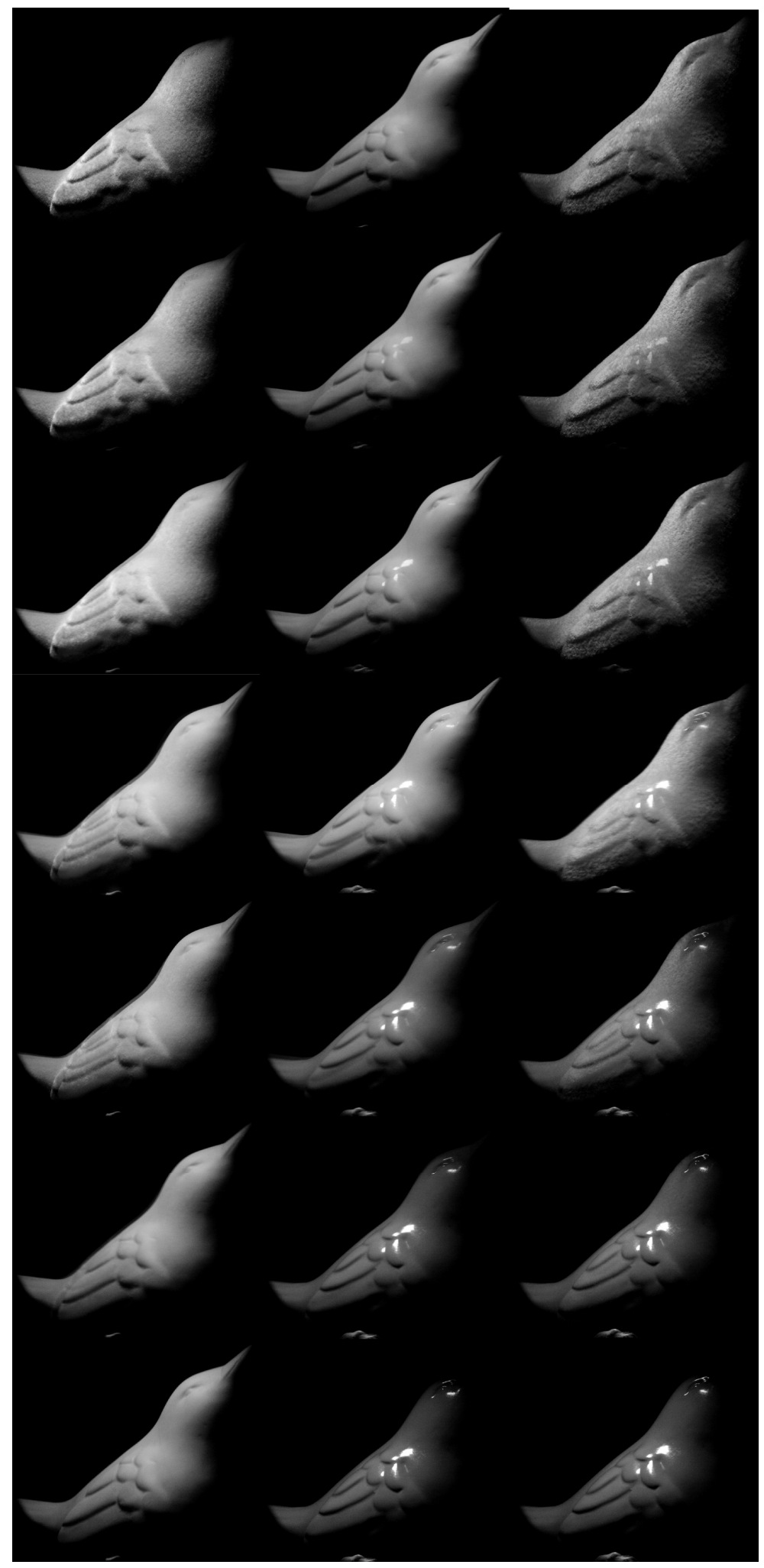

Figure 3. Photographs of the optical mixtures: in the left column velvety with matte, in the middle column matte with glossy and in the right column velvety with glossy. From top to bottom the $\log \left[\right.$ weight $_{1} /$ weight $\left._{2}\right]$ is $-2,-1,-.5$, $0,1, .5,1,2$, with contribution 1 the material dominating at the bottom and contribution 2 at the top. 


\section{Participants}

One female and seven male undergraduate students at the Delft University of Technology took part in the experiment. They received a compensation of 10 euro per hour for their participation. All participants were naive with respect to the setup of the experiment. They gave written, informed consent. All experiments were in agreement with local ethical guidelines, Dutch Law and the Declaration of Helsinki. All participants reported having normal or corrected-to-normal vision.

\section{Procedure}

We first showed a photograph of the glossy, matte and velvety birds (similar to Figure 2, but in color) to the participants. We explained that they had to judge the materials of which such birds were made. We explicitly asked them to judge the physical properties of the materials, and not judge the material in a metaphorical way. We also mentioned that the materials in the photograph were extreme cases of the stimuli that they were going to judge.

The eight participants made three repeated ratings for the 21 conditions in randomized block order. Block orders were balanced across participants. The ratings were collected using preprinted scales representing the matte-glossy, hard-soft, cold-warm and light-heavy dimensions on which the observers could indicate their rating by drawing a mark, see figure 4.

With each stimulus presentation they first viewed the stimulus for about 5 seconds. Next they started their ratings. If they needed another view of the stimulus they could again view the stimulus for 5 seconds, and so on, until they completed the four ratings of that stimulus.

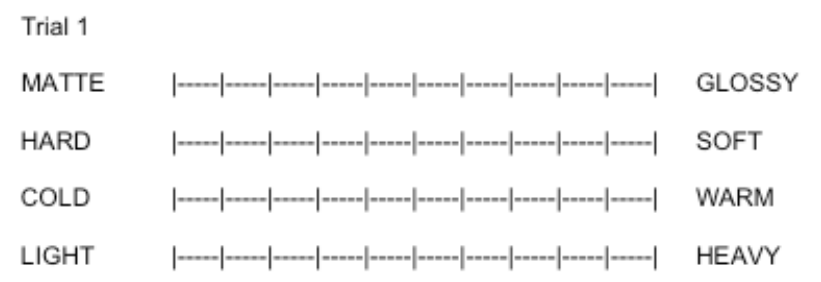

Figure 4. A reprint of the scales on the rating forms. Participants could indicate their rating by drawing a mark.

\subsection{Results}

We normalized the rating scales from 0 to 1 , with 0 (low scores) representing the most matte, hard, cold and light judgments and 1 (high scores) representing the most glossy, soft, warm and heavy judgments. We averaged the data over participants and repeated settings. Thus each data point in the following graphs represents 8 participants times 3 trials $=$ 24 settings per condition. We find very consistent results for the extreme (NDF 2) conditions, in which the object without the filter was extremely dominant: the bargraph in figure 5 shows these results as a function of combination (the grouped bars, above which we noted which material combination the specific groups correspond to). The graylevels represent the different ratings: glossiness, softness, warmth and heaviness from dark to light. In these extreme cases the primarily glossy bird was judged to be extremely glossy, very hard, very cold and rather heavy, the primarily matte bird was judged to be rather average on all scales, and the primarily velvety bird was rated to be very matte, soft, warm and rather light.

For the less extreme conditions (NDF $0,0.5$ and 1) we found the ratings to vary systematically as a function of the weights, see figure 6 . Here we show all average settings as a function of $\log [$ weight $/$ weight $]=\mathrm{NDF}_{1}-\mathrm{NDF}_{2}(\mathrm{see}$ figure 3). The different material combinations are represented in the columns, the rating properties in the rows. It is clear that the settings vary gradually as a function of the weightings. The largest range of the ratings was found for the glossiness settings (first row) and the smallest for heaviness judgments. 


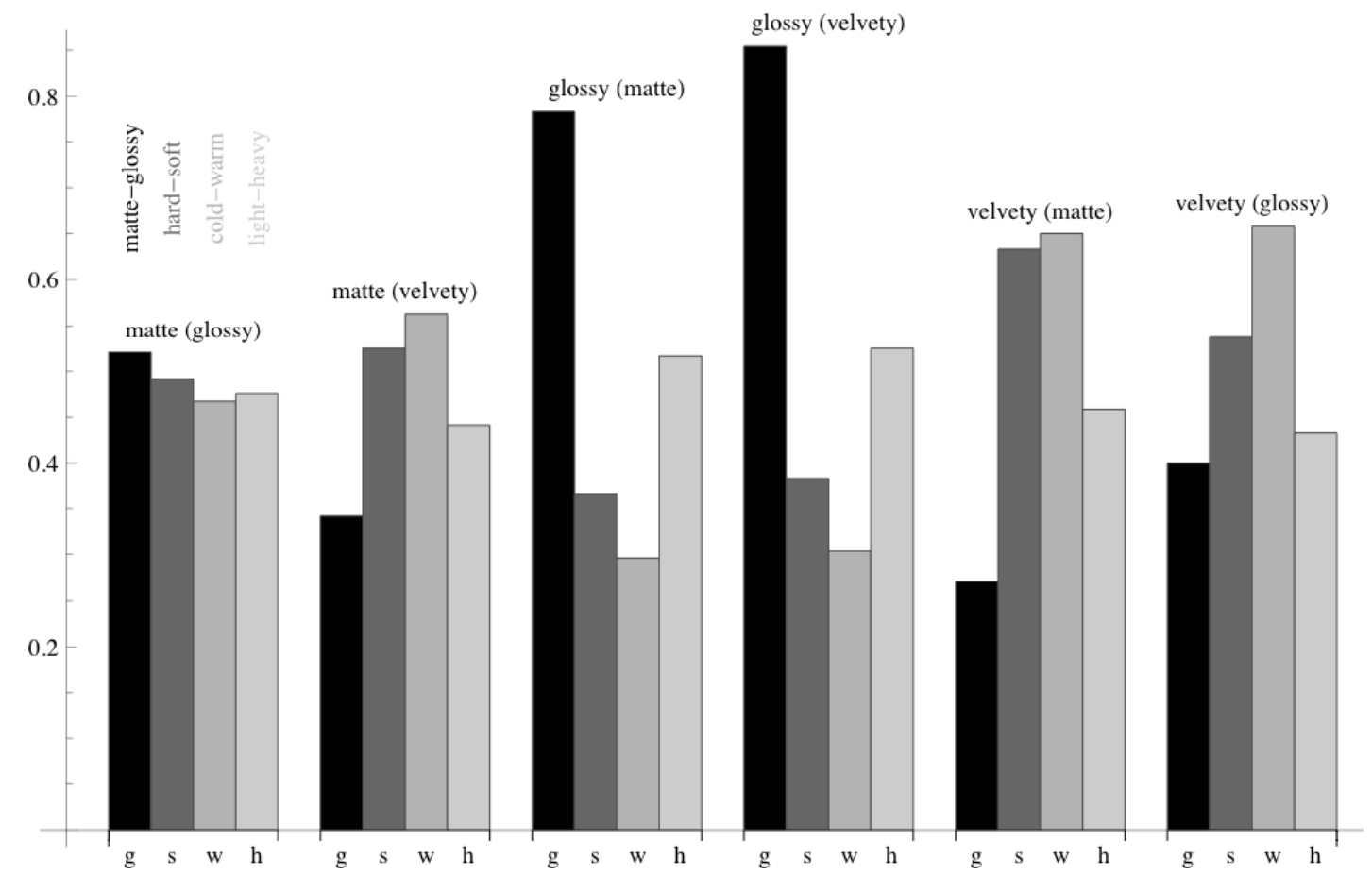

Figure 4. A bargraph of the average ratings (calculated over all participants and all three repeated settings) for the NDF 2 cases. Data were normalized to a range of 0-1with 0 (low scores) representing the most matte, hard, cold and light judgments and 1 (high scores) representing the most glossy, soft, warm and heavy judgments. The different graylevels depict the ratings for the different properties, in the order matte-glossy, hard-soft, cold-warm and lightheavy. They are grouped by the combination of birds for which the settings were done. Above the groups we noted for which combination of birds the grouped data were collected, with the first term denoting the dominant object and the second between brackets the severely (NDF 2, or a factor of 100) attenuated object.

\section{DISCUSSION}

We constructed a novel type of setup with which we can optically mix real stimuli. Clear advantages of this setup are that we can use real objects (so that dynamic range and resolution are not an issue and stereoscopic cues are present) and still vary their material properties in a very systematic way. Furthermore, the setup allows for a wide range of other possible manipulations, e.g. mixing objects and scenes / contexts and mixing different lighting settings. We are currently exploring various applications.

Superpositions of objects do not correspond to interpolated optical properties, such as linear combinations of BRDF's (e.g. matte plus shiny is assumed to represent glossy). This is due to interreflections ${ }^{7}$ (at the micro-, meso- and macroscales!). Thus, the optically mixed stimuli cannot simply be considered as physical mixes. Instead, they should be considered as "painterly mixes" of the most salient features of the two components, for instance white highlights on the glossy green bird, smooth shading over the matte green bird and white contours on the velvety bird (the main characteristic of asperity scattering ${ }^{3}$ ).

In the most extreme stimulus settings we still found an influence of the least weighted contribution. Note that in these cases the contributions differed by a factor of 100! For instance, a 100 times attenuated glossy bird mixed with a matte bird was rated differently than a 100 times attenuated velvety bird mixed with a matte bird. Thus, for some modes the main characteristics (e.g. highlights for glossy and bright contours for velvety) are so salient that they influence perception even if their optical influence is relatively very small. Data on such effects provide insights into relative contributions of optical material modes to perceived material qualities. We only studied small subsets of material modes and of perceptual properties and much more research is needed to get a good overview. 
We found robust and systematical results that vary according to the weights of our optical mixtures. Thus, our novel setup works as it is supposed to. Observers commented that they thought we actually presented birds made of varying materials and varying lighting. Another interesting finding is that our optical mixtures covary consistently in perceived glossiness, softness, warmth and heaviness, even though the latter three are properties that are not directly related to the optical properties.
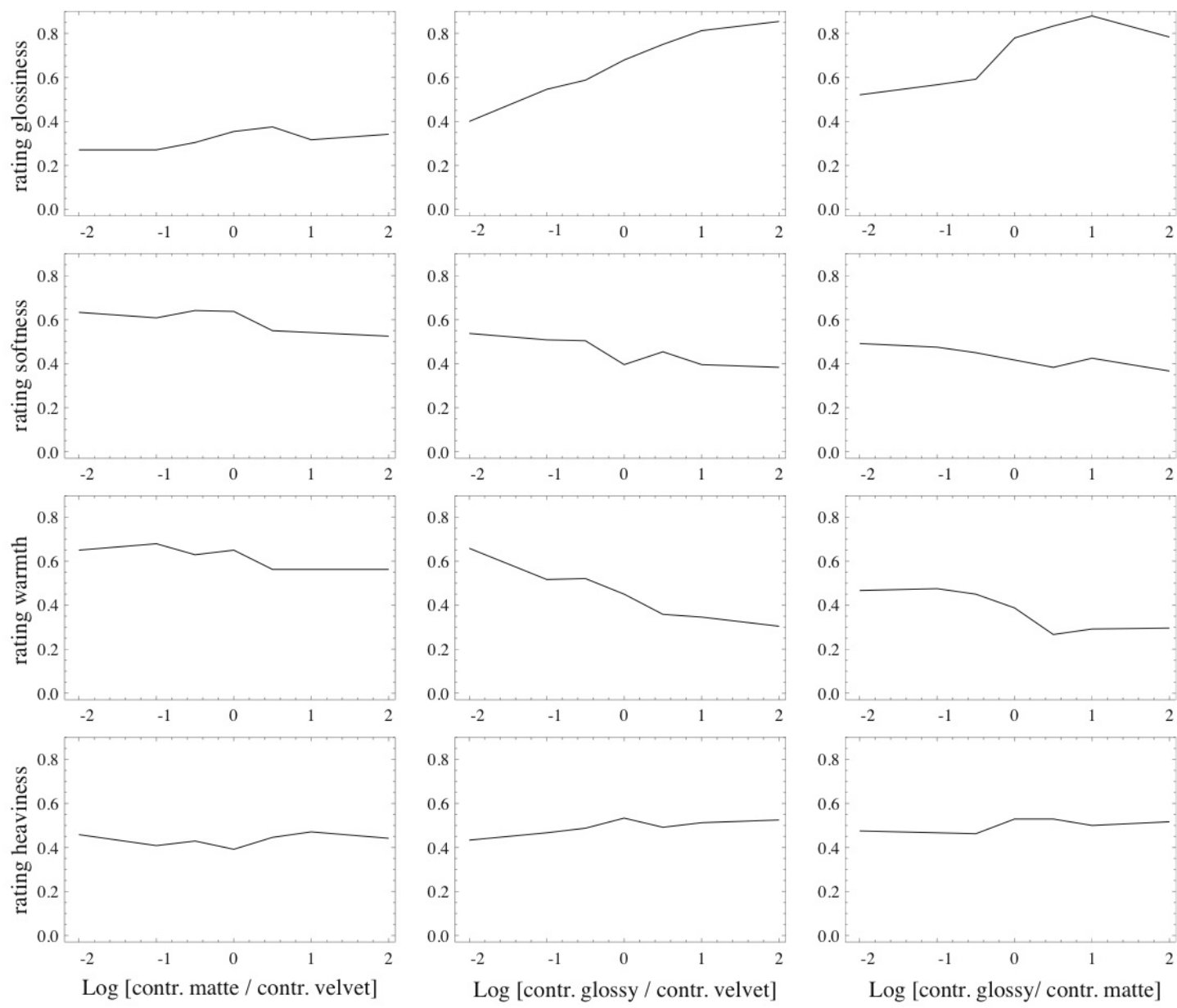

Figure 5. The average ratings (calculated over all participants and all three repeated settings, on a normalized range of $0-1)$ as a function of the logarithm of the ratio of two contributions of the optical mixtures. The left column depicts the results for the velvety-matte combination, the middle column for the velvety-glossy combination, and the right column for the matte-glossy combination. Thus, for instance, in the graphs in the left column the velvety contribution dominates at the left side and the matte contribution at the right side. The rows depict, from top to bottom, the ratings on the matte-glossy, hard-soft, cold-warm and light-heavy properties.

\section{ACKNOWLEDGEMENTS}

Maarten Wijntjes was supported by the Netherlands Organisation for Scientific Reserch (NWO). 


\section{REFERENCES}

[1] Dana, K. J., van Ginneken, B., "Reflectance and texture of real-world surfaces," Proceedings IEEE Computer Science Conference on Computer Vision and pattern Recognition (1977).

[2] Karana, E., Hekkert, P., Kandachar, P., "Meanings of Materials through sensorial properties and manufacturing processes," Materials and Design, 30, 2778-2784 (2009).

[3] Koenderink, J., J., Pont, S. C., "The secret of velvety skin," Machine Vision and Applications, special Issue on Human Modeling, Analysis and Synthesis, 14, 260-268 (2003).

[4] Koenderink, J., J., Pont, S. C., "Material properties for surface rendering," International Journal for Computational Vision and Biomechanics, 1(1), 43-53 (2008).

[5] Lambert, J.H., [Photometria Sive de Mensure de Gradibus Luminis, Colorum et Umbrae], Eberhard Klett, Augsburg (1760).

[6] Mausfeld, R., "The perception of material qualities and the internal semantics of the perceptual system," In: Albertazzi, L. and van Tonder, G. and Vishnawath, D., [Perception beyond Inference. The information Content of Visual Processes], MIT Press, Cambridge, Mass (2011).

[7] Pont, S., C., Koenderink, J. J., "Reflectance from locally glossy thoroughly pitted surfaces," Computer Vision and Image Understanding, 98, 211-222 (2005).

[8] Torrance, K., E., Sparrow, E. M., Birkebak, R. C., "Polarization, directional distribution, and off-specular peak phenomena in light reflected from roughened surfaces," Journal of the Optical Society of America A, 56(7), 916-925 (1966). 